\title{
Numerical solutions of the unsteady Fanno model for compressible pipe flow
}

\author{
A. NOVIKOVS, H. OCKENDON AND J. R. OCKENDON
}

OCIAM, Mathematical Institute, 24-29 St Giles', Oxford OX1 3LB, UK

(Received 1 September 2006 and in revised form 12 January 2007)

This paper presents numerical results on the evolution of the solutions of the Fanno model for compressible pipe flow. The principal results concern the large-time behaviour when nonlinear effects are appreciable throughout the evolution. Our computations show that compression waves can be expected to evolve into travelling waves for large times whereas expansion waves cannot.

\section{Introduction}

As described in Ockendon, Ockendon \& Falle (2001), the dimensionless Fanno model for turbulent gas flow in a rough pipe of constant cross-section in which the characteristic velocity, pressure and density are $u_{0}, p_{0}$ and $\rho_{0}$ is

$$
\begin{gathered}
\frac{\mathrm{d} \rho}{\mathrm{d} t}+\rho \frac{\partial u}{\partial x}=0, \\
\frac{\mathrm{d} u}{\mathrm{~d} t}+\frac{1}{\rho} \frac{\partial p}{\partial x}=-u|u|, \\
\frac{\mathrm{d}}{\mathrm{d} t}\left(\frac{p}{(\gamma-1) \rho}+\frac{1}{2} u^{2}\right)-\frac{1}{\rho^{2}}\left(\frac{1}{(\gamma-1) M_{0}^{2}}+p\right) \frac{\mathrm{d} \rho}{\mathrm{d} t}+\frac{u}{\rho} \frac{\partial p}{\partial x}=0,
\end{gathered}
$$

where $\gamma>1$ is the ratio of specific heats, $\mathrm{d} / \mathrm{d} t=\partial / \partial t+u \partial / \partial x$ and $M_{0}=u_{0} / a_{0}$, where $a_{0}^{2}=\gamma p_{0} / \rho_{0}$; the term involving $M_{0}$ in (1.3) appears because the difference between the pressure and $p_{0}$ has been non-dimensionalized with $\rho_{0} u_{0}^{2}$. This model only differs from the inviscid model in the right-hand side of (1.2), which represents the effect of friction at the pipe wall. However, while the characteristics are unaffected by the presence of this term, it does render the flow non-isentropic and there are no Riemann invariants.

The model can be validated from the mean turbulent flow equations for twodimensional flow in a thin channel with velocity vector $(u, v)$ as derived in Cebeci \& Smith (1974):

$$
\begin{gathered}
\frac{\partial \bar{\rho}}{\partial t}+\frac{\partial}{\partial x}(\bar{\rho} \bar{u})+\frac{\partial}{\partial y}(\overline{\rho v})=0 \\
\frac{\partial}{\partial t}(\bar{\rho} \bar{u})+\frac{\partial}{\partial x}(\bar{\rho} \bar{u} \bar{u})+\frac{\partial}{\partial y}(\overline{\rho v} \bar{u})+\frac{\partial \bar{p}}{\partial x}=\frac{\partial \bar{\tau}}{\partial y} \\
\frac{\partial}{\partial t}\left(\frac{\bar{p}}{\gamma-1}+\bar{\rho} \frac{\bar{u}^{2}}{2}\right)+\frac{\partial}{\partial x}\left(\bar{u}\left(\frac{\gamma \bar{p}}{\gamma-1}+\bar{\rho} \frac{\bar{u}^{2}}{2}\right)\right)+\frac{\partial}{\partial y}\left(\overline{\rho v}\left(\frac{\gamma \bar{p}}{(\gamma-1) \bar{\rho}}+\frac{\bar{u}^{2}}{2}\right)\right)=\frac{\partial}{\partial y}(\bar{u} \bar{\tau}),
\end{gathered}
$$


where the barred quantities are time-averaged over the largest turbulent time scale. The assumption that the flow is nearly unidirectional allows the stress, which comprises both viscous and Reynolds stresses, to be approximated by $\bar{\tau}$ and also allows approximations such as $\overline{\rho u}=\bar{\rho} \bar{u}$, whereas $\overline{\rho v} \neq \bar{\rho} \bar{v}$.

Assuming the channel is of constant width $l$, we integrate equations (1.4)-(1.6) with respect to $y$ from $-l / 2$ to $l / 2$ using no-slip boundary conditions. Setting $\int_{-l / 2}^{l / 2} \bar{u} \mathrm{~d} y=l u$ etc., and $\bar{\tau}= \pm \tau_{w}$ at the channel walls $\pm l / 2$, we obtain the dimensional form of the Fanno flow equations (1.1)-(1.3) under the assumption that the dimensional turbulent shear stress at the wall is $\tau_{w}=-\frac{1}{2} \rho u|u| f$. This formula was discussed extensively in the famous experimental work of Nikuradse (1950). There he elucidated how the friction factor $f$ at the wall of a pipe in which the flow is turbulent depends on the wall roughness and the Reynolds number $R e=U l / v$, where $U$ is the typical velocity of fluid, $v$ is the kinematic viscosity. These studies were recently confirmed theoretically by Gioia \& Chakraborty (2006). They predict that, for large $R e$, the friction factor is a function of roughness only and, for many commonly occurring rough pipes, $f \sim O\left(10^{-3}\right)$. However, we emphasize that all this experimental evidence only underpins the Fanno model for steady flow and it is merely a reasonable hypothesis for flows that are unsteady on a sufficiently long time scale.

The main aim of this paper is to present a reliable numerical procedure for solving (1.1)-(1.3) in two situations where there is analytical evidence for at least some of the features of the solution.

One class of problems to be addressed concerns flow in a semi-infinite pipe when the end pressure is suddenly increased or decreased. For small pressure changes relative to ambient, characterized by a dimensionless parameter $\epsilon \ll 1$, these problems are susceptible to asymptotic analysis, as described in Ockendon et al. (2001). The following temporal sequence emerges:

(i) for dimensional times of $O\left(l / a_{0} f\right)$ friction is negligible to lowest order;

(ii) for times of $O\left(l / \epsilon a_{0} f\right)$, the velocity is governed by a nonlinear damped wave equation of the form

$$
\frac{\partial^{2} u}{\partial t^{2}}-\frac{\partial^{2} u}{\partial x^{2}}=-2|u| \frac{\partial u}{\partial t}
$$

in suitable variables;

(iii) for even larger times of $O\left(l / \epsilon^{2} a_{0} f\right)$, the flow lags further and further behind the leading characteristic propagating from the end of the pipe, and the velocity satisfies the parabolic equation

$$
\frac{\partial^{2} u}{\partial x^{2}}=2|u| \frac{\partial u}{\partial t},
$$

again in suitable variables.

Another class of problems concerns piston motion in an infinitely long pipe, where asymptotic results are again available when the piston velocity is of $O\left(\epsilon a_{0}\right)$, where $\epsilon \ll 1$. The sequence of events is the same as for the pressure impulse problem, with the model becoming parabolic in nature over times of $O\left(l / \epsilon^{2} a_{0} f\right)$. However, for times of $O\left(l / \epsilon^{3} a_{0} f\right)$ or longer, the pressure and density variations grow to be of the order of their ambient values and the flow is then modelled by the degenerate hyperbolic system

$$
\frac{\partial \rho}{\partial t}+\frac{\partial}{\partial x}(\rho u)=0, \quad \frac{\partial p}{\partial x}+\gamma \rho u|u|=0, \quad \frac{\partial p}{\partial t}+\gamma \frac{\partial}{\partial x}(p u)=0 .
$$


(a)

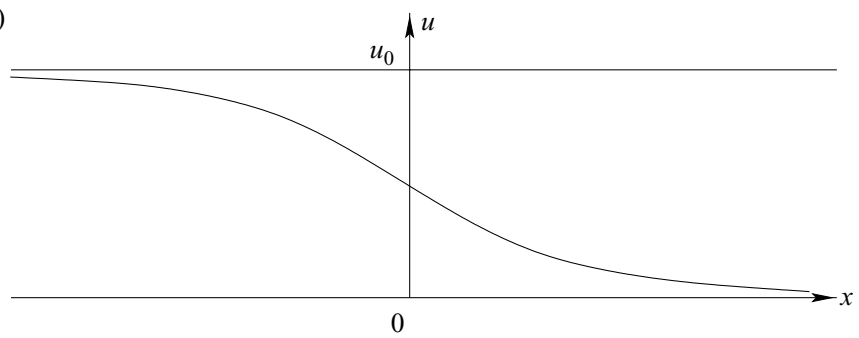

(b)

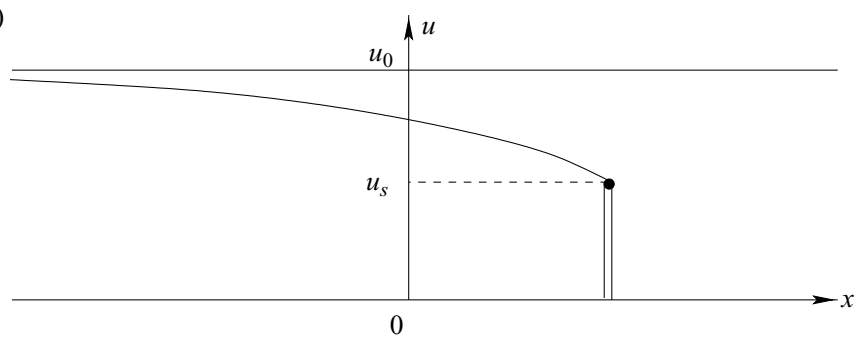

(c)

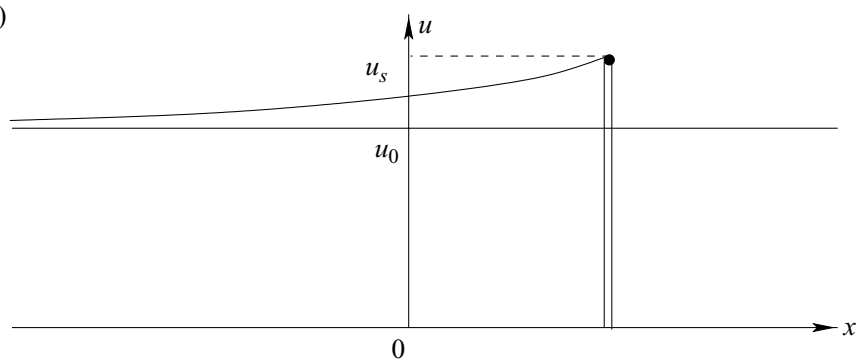

FIGURE 1. Piston problem: travelling wave solutions for different Mach number flows described in the text.

It was suggested in Ockendon et al. (2001) that a compressive piston motion eventually sets up a travelling wave in which a slug of gas moves with the piston velocity, the leading edge of this slug having a velocity $\gamma$ times the piston velocity; ahead of this slug, the gas is effectively at rest. For piston motions that expand the gas, a similar confinement occurs in the vicinity of the initial piston position, where a dissipative expansion wave describes the regime in which the flow is spatially varying.

These two situations will be analysed numerically in $\S 2$ and $\S 3$, in cases where $\epsilon$ is of $O(1)$ or smaller. One of our key results will be an assessment of another suggestion made in Ockendon et al. (2001), namely that there exists a solution of (1.1)-(1.3) which is a fully nonlinear wave of permanent form whose profile depends sensitively on the Mach number $M_{w}$ of the wave, defined as the ratio of the speed of the wave to the ambient sound speed ahead of the wave:

(i) if $M_{w}<1$, smooth compressive waves are possible with speed $\gamma u_{0}$, where $u_{0}$ is the speed behind the wave (figure $1 a$ );

(ii) if $1<M_{w}<\sqrt{2 \gamma /(\gamma-1)}$, the only possible travelling waves contain a shock, also moving with speed $\gamma u_{0}$ (figure $1 b$ );

(iii) if $M_{w}>\sqrt{2 \gamma /(\gamma-1)}$, scenario (ii) applies except that the velocity increases as the shock is approached from downstream (figure $1 c$ ). 
The only expansion wave of permanent form that was proposed in Ockendon et al. (2001) described the flow behind a withdrawn piston moving with velocity precisely $\gamma u_{0}$, where $u_{0}$ is the speed of the gas at $x=-\infty$. The validity of this scenario for travelling waves will be discussed in $\S 3$.

Since all the problems we will study have both initial and boundary conditions, our numerical procedure will use the characteristic-based method presented in Thompson $(1987,1990)$. The key idea of this method is to identify outgoing and incoming waves at the boundary and then satisfy the correct boundary conditions in terms of these waves. Moreover, since all the problems have a moving boundary, we will transform them into a Lagrangian frame $(\xi, t)$ where the boundary is fixed, at $\xi=0$.

To perform the characteristic analysis we need the primitive form of the hyperbolic system we study, namely

$$
\frac{\partial \boldsymbol{u}}{\partial t}+\boldsymbol{A} \frac{\partial \boldsymbol{u}}{\partial \xi}=\boldsymbol{c}
$$

and which also may be written as

$$
\boldsymbol{S} \frac{\partial u}{\partial t}+\boldsymbol{L}=\boldsymbol{S} c
$$

where the rows of $\boldsymbol{S}$ are the left eigenvectors $\boldsymbol{l}^{(i)}$ corresponding to the eigenvalues $\lambda^{(i)}$ of matrix $\boldsymbol{A}$; the components $L^{(i)}$ of vector $\boldsymbol{L}$ are defined as

$$
L^{(i)} \equiv \lambda^{(i)} \boldsymbol{l}^{(i)} \frac{\partial \boldsymbol{u}}{\partial \xi}
$$

The eigenvalues $\lambda^{(i)}$ are the slopes $\mathrm{d} \xi / \mathrm{d} t$ of the characteristics of the hyperbolic system (1.7), and (1.8) are differential relations along the characteristics. Depending on the direction of the characteristics at the boundary these relations represent incoming or outgoing waves $\dagger$. In particular, for problems posed in a semi-infinite region $\xi \geqslant 0$, the incoming waves at $\xi=0$ are those with $\lambda^{(i)}<0$ and they carry information from earlier times. Thus, as long as the boundary is not a characteristic, the variables associated with incoming waves can be found numerically using the definition of $L^{(i)}$ in (1.9). The numerical approximation of (1.8) will be based on one-sided finite difference approximations involving only interior and boundary points, $\xi \geqslant 0$.

We will also encounter Goursat problems, when the boundary coincides with one of the characteristic paths, along which (1.8) becomes an ordinary differential equation in time. In this case, the outgoing waves with $\lambda^{(i)}>0$ at $\xi=0$ depend on data on the boundary. For these waves $L^{(i)}$ have to be found from (1.8) using the given boundary conditions at $\xi=0$.

In any case, the problem of implementing boundary conditions is reduced to the problem of computing the appropriate values for the $L^{(i)}$ terms in (1.8)-(1.9). Once all the boundary values are computed, it is possible to use higher-order finite difference approximations for (1.8) in the interior of the domain when $\xi>0$ to improve the global convergence.

$\dagger$ The words incoming and outgoing do not have universal interpretations in the literature. By incoming we will mean a wave corresponding to a characteristic that impinges the boundary from earlier times from within the field of flow. 
(a)

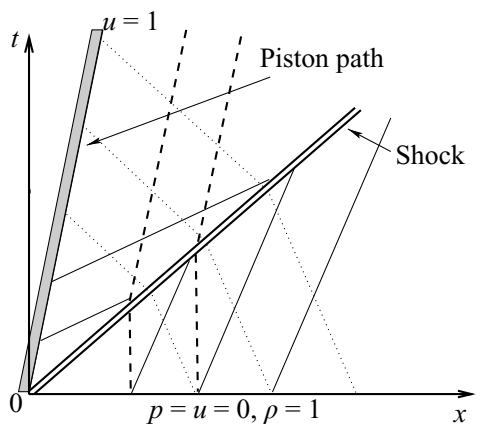

(b)

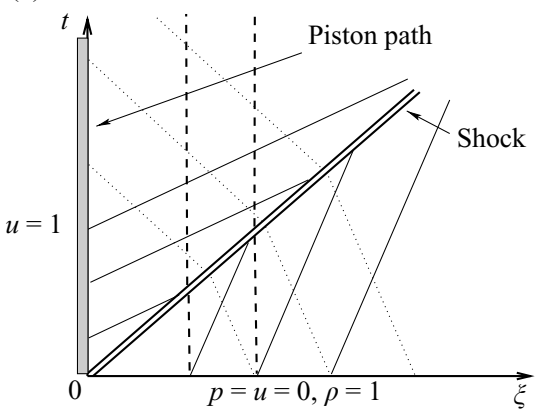

FiguRE 2. The characteristic structure for the compressive piston problem: —, positive characteristics; $\cdots$, negative characteristics; ---, particle path. (a) Fixed space coordinate, (b) Lagrangian frame.

\section{Piston problems}

\subsection{Compressive waves}

We consider a piston moved impulsively with constant non-dimensional velocity $u=1$ into a quiescent gas occupying $x \geqslant 0$, so that initial conditions are

$$
u=p=0, \quad \rho=1 \quad \text { in } x>0,
$$

and $u=1$ at the piston $x=t$ for all $t>0$. Therefore we can also assume non-negative velocity $u$ throughout the flow.

The particle path $\mathrm{d} x / \mathrm{d} t=u$ is one of the characteristics of the Fanno model (1.1)-(1.3), and the one that starts at $t=0, x=0$ coincides with the piston path as shown in figure 2(a). Thus, we have a well-posed Goursat problem. To transform the moving-boundary value problem into a problem with fixed boundary we introduce a Lagrangian coordinate

$$
\xi=\int_{x(t)}^{x} \rho(s, t) \mathrm{d} s
$$

where $x(t)$ is a particle path. Then equations (1.1)-(1.3) can be transformed into the conservative Lagrangian form

$$
\begin{gathered}
\frac{\partial}{\partial t}\left(\frac{1}{\rho}\right)-\frac{\partial u}{\partial \xi}=0, \\
\frac{\partial u}{\partial t}+\frac{\partial p}{\partial \xi}=-u^{2}, \\
\frac{\partial e}{\partial t}+\frac{\partial}{\partial \xi}\left(u\left(p+\frac{1}{(\gamma-1) M_{0}^{2}}\right)\right)=0,
\end{gathered}
$$

where $e=p /(\gamma-1) \rho+u^{2} / 2$; in primitive variables the energy equation (2.5) becomes

$$
\frac{\partial p}{\partial t}+\alpha^{2} \frac{\partial u}{\partial \xi}=(\gamma-1) \rho u^{3},
$$

where $\alpha^{2}=\gamma p \rho+\rho / M_{0}^{2}$. It is not difficult to show by using the chain rule and product rule the equivalence of the Euler and Lagrangian equations for classical solutions. Also, Wagner (1987), using techniques from measure theory, has proved that there is a one-to-one correspondence between bounded and measurable weak solutions of 
the Euler equations and weak solutions for the same problem written in Lagrangian coordinates.

The initial conditions in the moving frame become

$$
u=p=0, \quad \rho=1 \quad \text { in } \xi>0 \text { at } t=0,
$$

while, at the boundary $\xi=0$, we have $u=1$ for $t>0$. The inverse transformation of the Lagrangian coordinate is

$$
x=x_{0}(t)+\int_{0}^{\xi} 1 / \rho(s, t) \mathrm{d} s
$$

and $x_{0}(t)$ satisfies the equation

$$
\frac{\mathrm{d} x_{0}}{\mathrm{~d} t}=u(0, t)
$$

In our case the piston path is known and therefore $x_{0}(t)=t$.

For (2.3)-(2.4) and (2.6) the eigenvalues are

$$
\lambda^{(1)}=-\alpha, \quad \lambda^{(2)}=0, \quad \lambda^{(3)}=\alpha,
$$

and the corresponding left eigenvectors are

$$
\boldsymbol{l}^{(1)}=[0,-\alpha, 1], \quad \boldsymbol{l}^{(2)}=\left[\alpha^{2}, 0,1\right], \quad \boldsymbol{l}^{(3)}=[0, \alpha, 1] .
$$

From (1.9) with $\boldsymbol{u}=[1 / \rho, u, p]^{T}$ we obtain

$$
L^{(1)}=\lambda^{(1)}\left(-\alpha \frac{\partial u}{\partial \xi}+\frac{\partial p}{\partial \xi}\right), \quad L^{(2)}=0, \quad L^{(3)}=\lambda^{(3)}\left(\alpha \frac{\partial u}{\partial \xi}+\frac{\partial p}{\partial \xi}\right) .
$$

After inverting these definitions and substituting for $\partial \boldsymbol{u} / \partial \xi$ in terms of $\boldsymbol{L}$ in (2.3)-(2.4), (2.6) we end up with the primitive equations (1.8), written as

$$
\left.\begin{array}{l}
\frac{\partial}{\partial t}\left(\frac{1}{\rho}\right)-\frac{1}{2 \alpha^{2}}\left(L^{(3)}+L^{(1)}\right)=0, \\
\frac{\partial u}{\partial t}+\frac{1}{2 \alpha}\left(L^{(3)}-L^{(1)}\right)=-u^{2}, \\
\frac{\partial p}{\partial t}+\frac{1}{2}\left(L^{(3)}+L^{(1)}\right)=(\gamma-1) \rho u^{3} .
\end{array}\right\}
$$

Since $\lambda^{(1)}$ is always negative, it corresponds to an incoming characteristic at the boundary $\xi=0$ as in figure $2(b)$. Therefore, the corresponding value of $L^{(1)}$ has to be computed from its definition in (1.9) using the one-sided difference approximation

$$
\frac{\partial}{\partial \xi} \boldsymbol{u}_{0, n}=\frac{1}{\Delta \xi}\left(\boldsymbol{u}_{1, n}-\boldsymbol{u}_{0, n}\right)+O(\Delta \xi) \text { for } n \geqslant 1,
$$

the computational domain being defined as $\left(\xi_{j}, t_{n}\right) \equiv(j \Delta \xi, n \Delta t), j \in[0, N]$. The characteristic corresponding to $\lambda^{(3)}$ is outgoing from the boundary $\xi=0$ and the value of $L^{(3)}$ is specified from the boundary condition $\partial u / \partial t=0$ at $\xi=0$. This condition is satisfied if $L^{(3)}=L^{(1)}-2 \alpha$, which, together with (2.12), gives the pressure and density at the piston. To approximate the time derivative at the boundary we use the one-step forward Euler method.

For the numerical solution of the interior problem $(j, n \geqslant 1)$ we will solve the conservative Lagrangian system (2.3)-(2.5), thereby ensuring the approximate satisfaction of the Rankine-Hugoniot conditions for any shock that appears. The eigenvalues of 
this system are again given by (2.9) but the left eigenvectors are now

$$
\left.\begin{array}{l}
\tilde{\boldsymbol{l}}^{(1)}=[p /(\gamma-1), u+\alpha /(\gamma-1) \rho,-1], \\
\tilde{\boldsymbol{l}}^{(2)}=\left[p+1 /(\gamma-1) M_{0}^{2},-u, 1\right], \\
\tilde{\boldsymbol{l}}^{(3)}=[p /(\gamma-1), u-\alpha /(\gamma-1) \rho,-1] .
\end{array}\right\}
$$

After multiplying equations $(2.3)-(2.5)$ by the matrix $\tilde{\boldsymbol{S}}$ of left eigenvectors $\tilde{\boldsymbol{l}}^{(i)}$, the relations along the characteristics are

$$
\tilde{\boldsymbol{S}} \frac{\partial \boldsymbol{u}}{\partial t}+\tilde{\boldsymbol{S}} \frac{\partial}{\partial \xi} \boldsymbol{f}(\boldsymbol{u})=\tilde{\boldsymbol{S}} \boldsymbol{c}
$$

with

$$
\boldsymbol{u}=[1 / \rho, u, e]^{T}, \quad \boldsymbol{f}(\boldsymbol{u})=\left[-u, p, u\left(p+1 /(\gamma-1) M_{0}^{2}\right)\right]^{T}, \quad \boldsymbol{c}=\left[0,-u^{2}, 0\right]^{T} .
$$

Since $\lambda^{(3)}$ is always positive, we use a first-order upwind scheme to approximate the spatial derivative in the corresponding component of equation (2.15). Similarly, we use a one-step downwinding stencil in the equation along the negative characteristic corresponding to $\lambda^{(1)}$. For the time discretization, the forward Euler method again will be applied. Rewriting (2.15) in a discrete form, we then need to solve

$$
\begin{aligned}
& \tilde{\boldsymbol{l}}_{j, n}^{(1)} \mathscr{L} \boldsymbol{u}_{j, n}=-\tilde{\boldsymbol{l}}_{j, n}^{(1)} \mathscr{L}^{+} \boldsymbol{f}\left(\boldsymbol{u}_{j, n}\right)-u_{j, n}^{2}\left(u_{j, n}-\frac{\lambda_{j, n}^{(1)}}{(\gamma-1) \rho_{j, n}}\right), \\
& \tilde{\boldsymbol{l}}_{j, n}^{(2)} \mathscr{L} \boldsymbol{u}_{j, n}=u_{j, n}^{3}, \\
& \tilde{\boldsymbol{l}}_{j, n}^{(3)} \mathscr{L} \boldsymbol{u}_{j, n}=-\tilde{\boldsymbol{l}}_{j, n}^{(3)} \mathscr{L}^{-} \boldsymbol{f}\left(\boldsymbol{u}_{j, n}\right)-u_{j, n}^{2}\left(u_{j, n}-\frac{\lambda_{j, n}^{(3)}}{(\gamma-1) \rho_{j, n}}\right),
\end{aligned}
$$

for $j, n \geqslant 1$; the time step $\Delta t$ is chosen to satisfy the CFL condition that the characteristic through a point must lie within the domain of dependence at that point, and the finite difference operators are defined as

$$
\mathscr{L} \boldsymbol{u}=\frac{\boldsymbol{u}_{j, n+1}-\boldsymbol{u}_{j, n}}{\Delta t}, \quad \mathscr{L}^{+} \boldsymbol{u}=\frac{\boldsymbol{u}_{j+1, n}-\boldsymbol{u}_{j, n}}{\Delta \xi}, \quad \mathscr{L}^{-} \boldsymbol{u}=\frac{\boldsymbol{u}_{j, n}-\boldsymbol{u}_{j-1, n}}{\Delta \xi} .
$$

The above discretization has been chosen because of its ability to predict large-time solutions to Fanno problems. Unfortunately, this scheme is not fully conservative and numerical dissipation may be expected when strong shocks occur. To check this possibility, we have tested the code against exact solutions for piston problems without the Fanno term. The results are shown in figure 3, which gives us confidence that even when the piston Mach number is $M_{0}=1.4$, the error in the shock position at $t=100$ is still less than $5 \%$.

All the numerical computations were performed on a grid of length 120, divided into 1600 subintervals $(N=1600, \Delta \xi=0.075)$. The ratio of specific heats $\gamma$ was taken to be 1.4 throughout. For the first test, in the light of the predictions in Ockendon et al. (2001), the Mach number is small enough to satisfy $M_{w}=\gamma M_{0}<1$, so we choose $M_{0}=\epsilon=0.1$. In this case we expect a smooth travelling wave solution to exist when time becomes $t \geqslant O\left(\epsilon^{-2}\right)$. Figure 4 shows the comparison of the numerical and asymptotic solutions when $t=O\left(\epsilon^{-3}\right)$; this situation was also indicated schematically in figure $1(a)$.

In the hope of obtaining a travelling wave solution as in figure $1(b)$, we next choose the Mach number $M_{0}$ to be in the interval $(1 / \gamma, \sqrt{2 / \gamma(\gamma-1)})$. The computed velocity 


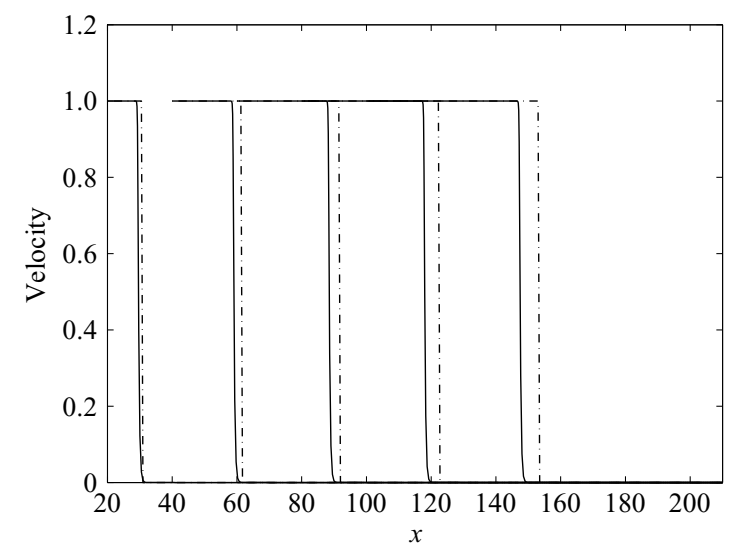

FIGURE 3. Comparison of numerical (solid line) with exact (dot-dashed line) solution of inviscid gas flow for compressive piston motion at times $t=20,40,60,80,100$, and $M_{0}=1.4$.

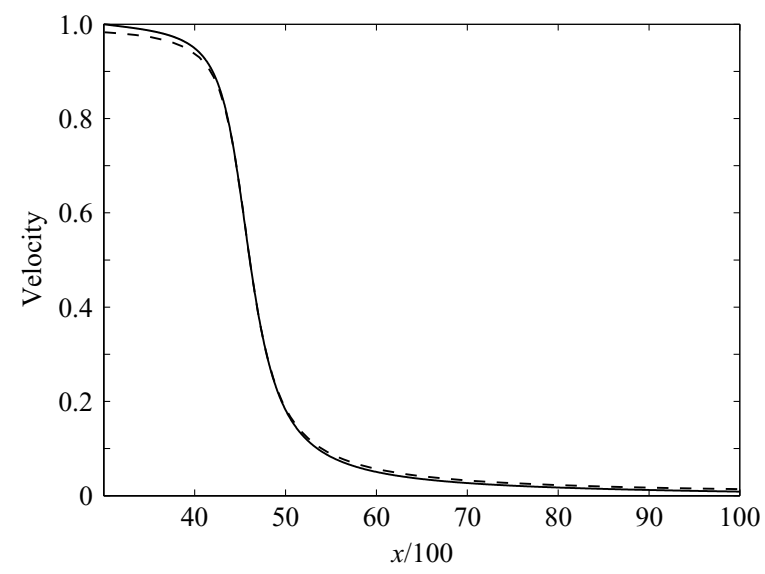

FIGURE 4. Comparison of numerical (solid line) with asymptotic (dashed line) solution for compressive piston motion when $t=3000$ and $M_{0}=\epsilon=0.1$.

profiles at different time steps and the asymptotic solution are shown in figure $5(a, b)$ when $M_{0}=1.4$. We observe a shock, which was correctly captured by the numerical scheme, moving at the predicted speed $\gamma$.

For even larger Mach numbers with $M_{0} \geqslant \sqrt{2 / \gamma(\gamma-1)}$, travelling wave solutions also exist, as in figure $1(c)$. The solutions for $u$ at $t=20,40,60,80,100$ and $M_{0}=3.5$ are shown in figure $5(c, d)$. As expected, the velocity grows spatially until it reaches the constant velocity $u_{s}$ at the shock position $x=\gamma t$. Figure $5(d)$ shows the comparison between the numerical and asymptotic solutions near the shock. The numerical solution gives $u_{s}=1.1189$ in comparison with the predicted value $(2 \gamma / \gamma+1)(1-$ $\left.1 / \gamma^{2} M_{0}^{2}\right)=1.1181$ from Ockendon et al. (2001).

We also present the corresponding pressure profiles when $M_{0}=1.4$ and $M_{0}=3.5$ in figure 6 in order to emphasize the strong qualitative difference between inviscid shocks and shocks in the Fanno model. 

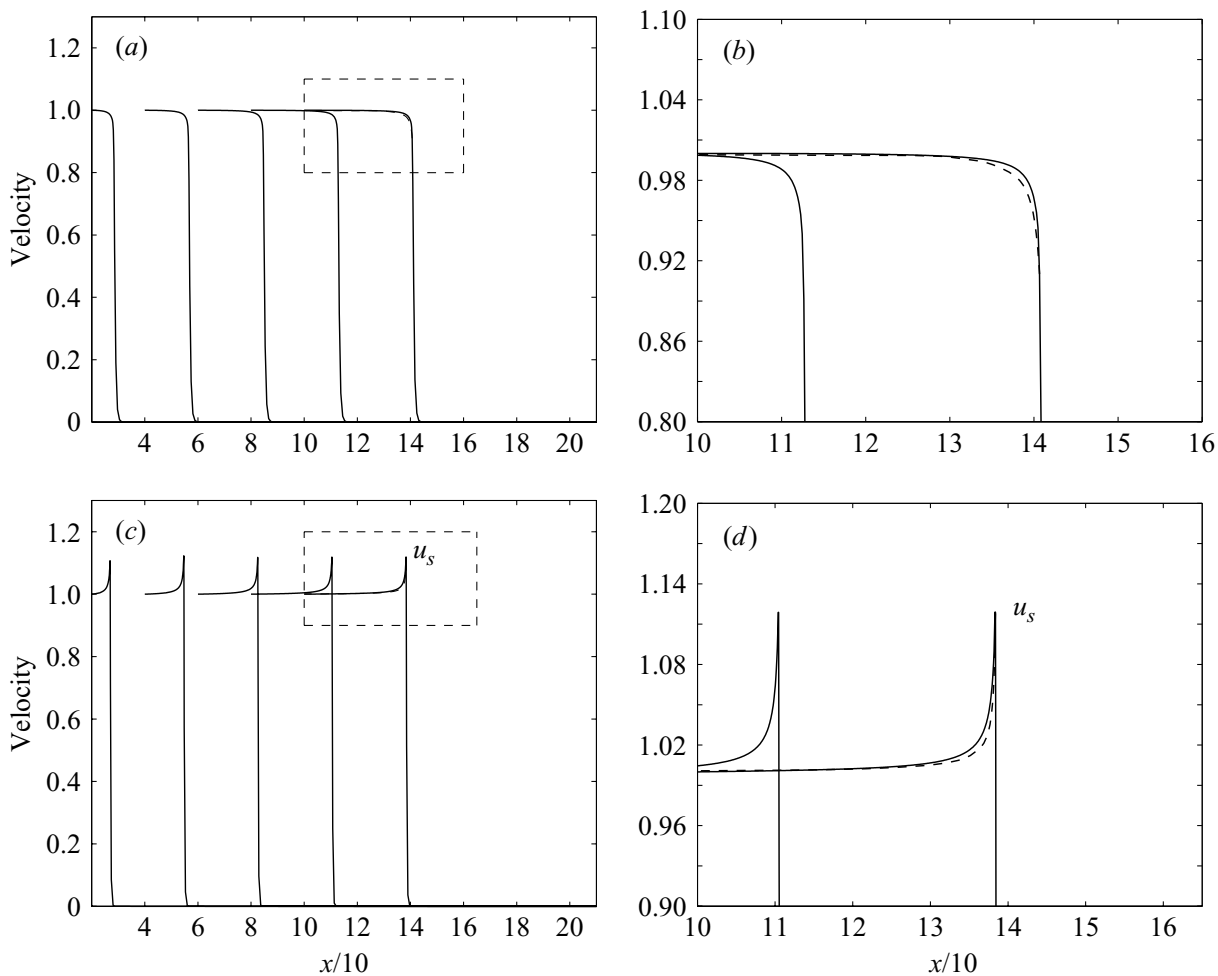

FIGURE 5. Numerical (solid line) and asymptotic (dashed line) solutions for the compressive piston problem when $(a, b) M_{0}=1.4$ and $(c, d) M_{0}=3.5$. Computed velocity profiles at times $t=20,40,60,80,100$, and asymptotic velocity profile at $t=100$. (b) Magnification of $(a)$ in the range $x \in[100,160]$. (d) Magnification of $(c)$ in the range $x \in[100,165]$.
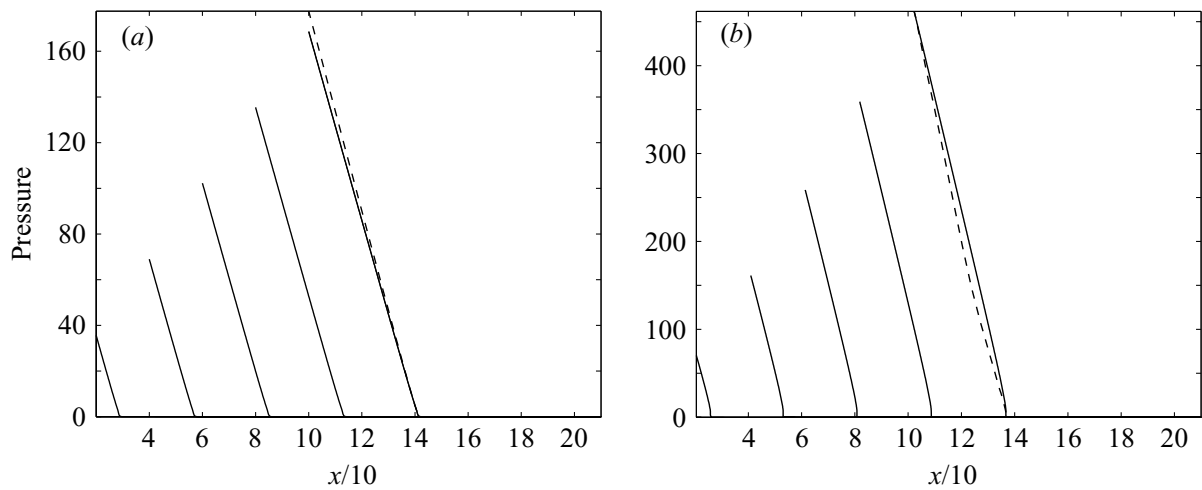

FIGURE 6. Computed pressure profiles (solid line) for the compressive piston problem at times $t=20,40,60,80,100$ for different Mach numbers: $(a) M_{0}=1.4,(b) M_{0}=3.5$. The comparison with asymptotic solutions (dashed line) corresponds to $t=100$.

\subsection{Expansion waves}

Now we suppose that gas initially occupies the region $x<0$ but flows in the positive $x$-direction behind a piston at $x=t$. In this case we again can assume that the velocity of the gas $u$ is non-negative throughout the flow. As in the compression case 

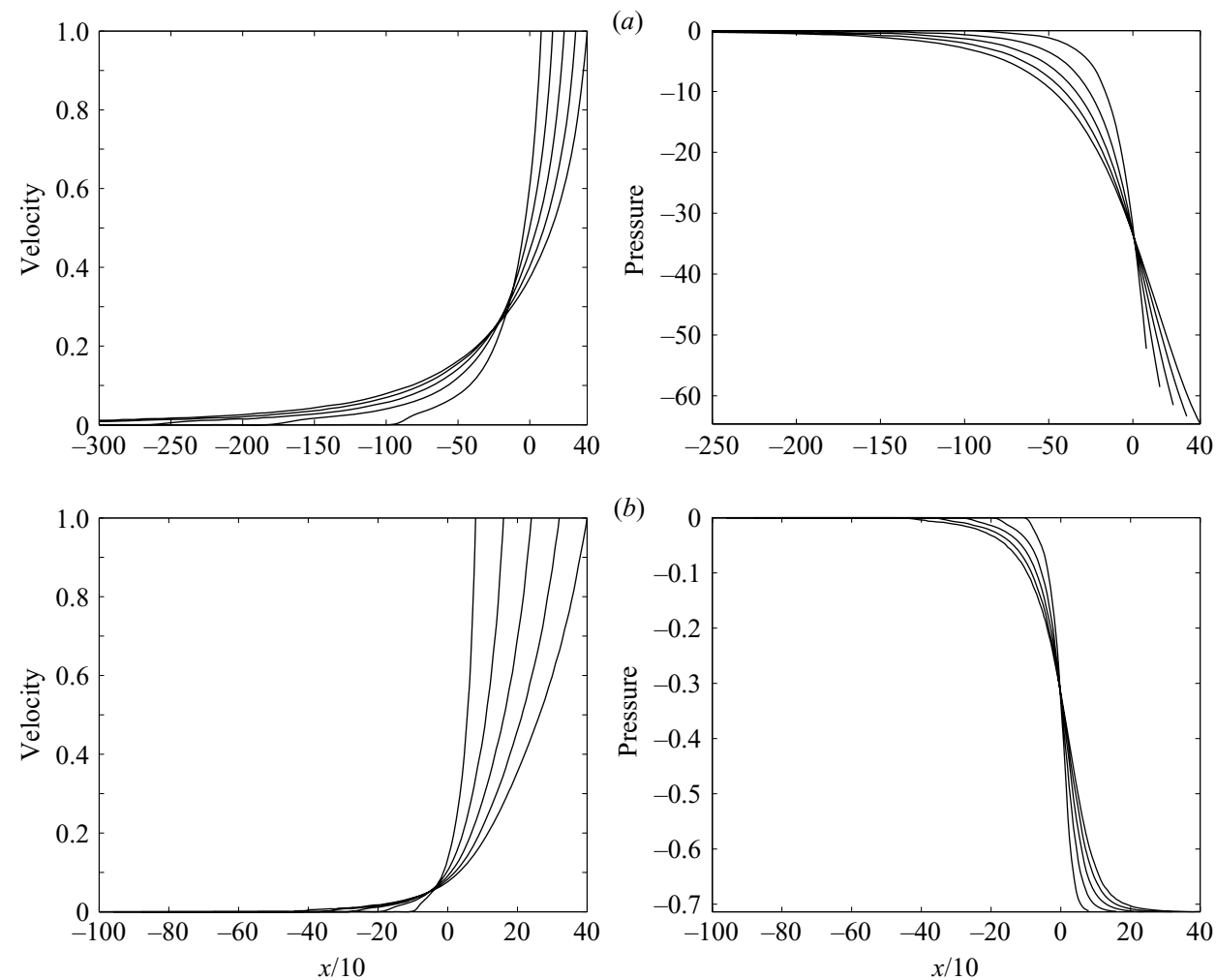

FIGURE 7. Velocity and pressure profiles of the expanding piston problem at times $t=80,160$, 240, 320, 400 for different Mach number flows: (a) $M_{0}=0.1$, (b) $M_{0}=1$. Note, that both velocity and pressure profiles become less steep as time increases.

we transform the problem into a Lagrangian frame in which the initial and boundary conditions are

$$
\begin{array}{ll}
u=p=0, & \rho=1 \quad \text { in } \quad \xi<0 \quad \text { at } t=0, \\
u=1 \quad \text { in } t>0 \quad \text { at } \quad \xi=0 .
\end{array}
$$

After the withdrawal of the piston, expansion waves start propagating in the region $\xi<0$ and these are associated with the characteristics of negative slope. At the piston $\xi=0$, the positive characteristics represent incoming waves, so the characteristicbased boundary conditions become different from those in compressive piston case. In particular, the value of $L^{(3)}$ at $\xi=0$ must be computed from its definition in (1.9) using only the interior and boundary points of the computational domain defined as $\left(\xi_{j}, t_{n}\right) \equiv(\Delta \xi(j-N), n \Delta t), j \in[0, N]$. From (2.12) we see that the boundary condition $\partial u / \partial t=0$ at $\xi=0$ is satisfied if $L^{(1)}=L^{(3)}+2 \alpha$. Putting this back into (2.12), we can determine $1 / \rho$ and $p$ at the piston. To approximate the time derivative, we again use the forward Euler method and, to compute the interior problem when $j, n \geqslant 1$, the numerical procedure is the same as for compressive motion.

The computed velocities and pressures are shown in figure 7 at different times and for different Mach number flows. Note that none of these expansion waves appears to tend to a travelling wave for large times and we will discuss the reason for this in $\S 3$. 
(a)

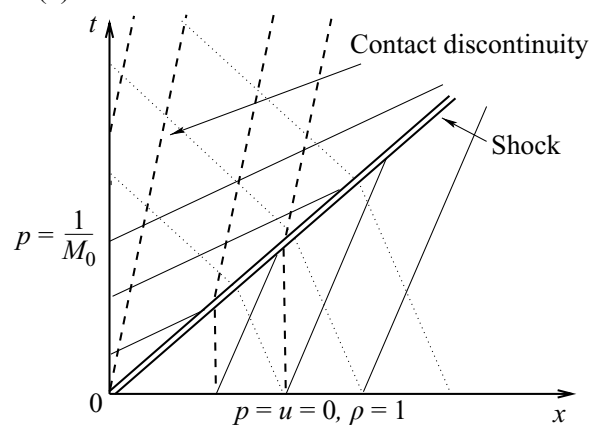

(b)

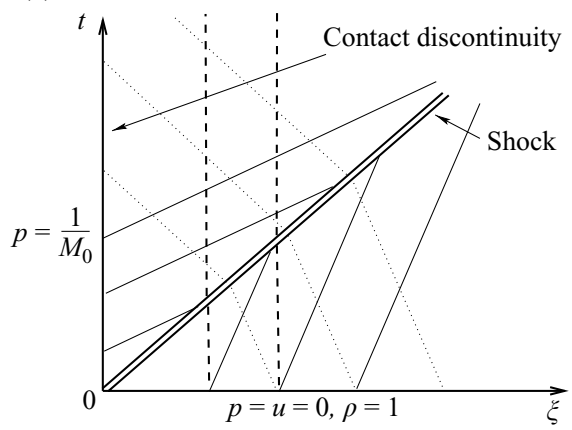

FIGURE 8 . The characteristic structure for the compressive pressure-jump problem:-, positive characteristics; $\cdots$, negative characteristics; ---, particle path. (a) Fixed space coordinate, (b) Lagrangian frame.

\section{Pressure-jump problems}

\subsection{Compressive waves}

In this section, we consider gas flow in a semi-infinite tube $x \geqslant 0$ produced by a sudden change in pressure at the end $x=0$, the gas being at rest with dimensional pressure $p_{0}$ and density $\rho_{0}$ at $t=0$. Such problems are motivated by the application of (1.1)-(1.3) to flows in pressure transducers and mine shafts (Mathematics in Industry Study Group 2005), which can be modelled by prescribing a pressure jump precisely at the end of the tube, $x=0$. However, the resulting characteristic diagram in figure $8(a)$ reveals that there are two outgoing characteristic families from the boundary $x=0$; they have slopes $\mu=\mathrm{d} x / \mathrm{d} t=u, u+c$, where $c=\sqrt{\gamma p / \rho}$ is the speed of sound. Therefore, we expect to prescribe two conditions on $x=0$ rather than just pressure to obtain well-posedeness. In practice, however, the end pressure will be controlled by gas flowing into or out of the pipe from the region $x<0$, so that it is realistic on physical grounds to impose the pressure on the gas particle path corresponding to $\mathrm{d} x / \mathrm{d} t=u$ with $x(0)=0$ rather than at the fixed boundary. In order to be fully realistic we would have to introduce a contact discontinuity $x=x_{c}(t)$ defined as $\mathrm{d} x_{c} / \mathrm{d} t=u$ with $x_{c}(0)=0$ and solve equations behind this discontinuity as well as ahead of it. Taking pressure to be constant on this particle path is equivalent to assuming the external flow comes from a large reservoir. We will discuss the implication of contact discontinuities in the conclusion.

With prescribed pressure on the initial particle path, the problem becomes a Goursat problem for which it is natural to introduce a material coordinate as in (2.2), leading to the non-dimensional Lagrangian equations (2.3)-(2.5). The initial conditions are

$$
u=p=0, \quad \rho=1 \quad \text { when } \quad \xi>0, t=0 .
$$

In order to write down the boundary condition, we must remember that the scalings leading to (1.1)-(1.3) correspond to dimensional pressures $p_{0}+\rho_{0} u_{0}^{2} p=p_{0}\left(1+\gamma M_{0}^{2} p\right)$. Moreover, in order to maintain consistency with our use of $M_{0}$ in $\S \S 1$ and 2, we write the dimensional pressure applied to the end of the tube as $p_{0}\left(1+\gamma M_{0}\right)$, which determines $M_{0}$ for this problem. Hence, even though larger dimensional applied pressures lead to larger Mach numbers, our non-dimensional boundary condition is

$$
p=\frac{1}{M_{0}} \quad \text { at } \quad \xi=0, \quad t>0 .
$$



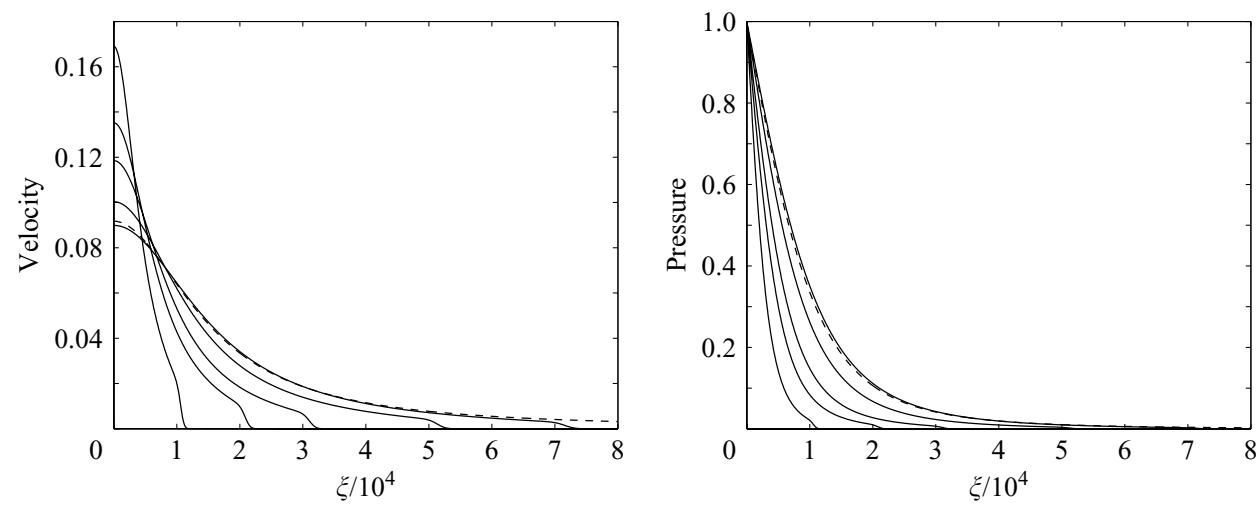

Figure 9. Computed velocity and pressure profiles (solid line) for the compressive pressure-jump problem at times $t=100,200,300,500,700$ when $M_{0}=0.01$; pressure is scaled with 0.01 . The comparison with asymptotic solutions (dashed line) corresponds to $t=700$.

As in (2.13), the structure of characteristics at the boundary $\xi=0$ suggests the use of a first-order downwind scheme to approximate spatial derivatives in the definition of $L^{(1)}$ in $(2.11)$, see figure $8(b)$. At the boundary $\xi=0$, the condition is $\partial p / \partial t=0$, which implies $L^{(3)}=-L^{(1)}+2(\gamma-1) u^{3} \rho$. Having computed $L^{(1)}$ and $L^{(3)}$ in the primitive equations (2.12) we can find $u$ and $\rho$ on $\xi=0$ for all $t>0$. To find the solution inside the domain $\xi, t>0$ we use the same method as for the piston problems in $\S 2$.

Figure 9 compares the numerical and asymptotic solutions for $M_{0}=0.01$ when time has reached $t=O\left(M_{0}^{-2}\right)$. We see good agreement between the velocities and pressures as expected for $t \geqslant O\left(M_{0}^{-1}\right)$. For Mach number of $O(1)$ and larger the velocity and pressure profiles at different time steps are plotted in figure 10. This figure contrasts strongly with figure 6 because, in the pressure-jump problems, there is no mechanism generating the high pressures associated with 'Fanno shocks'.

\subsection{Expansion waves}

Now we consider the case when expansion waves occur due to a sudden pressure drop at one end of the tube. The initial conditions are still (3.1) but, at the boundary $x=0$, we have a sudden decrease in pressure

$$
p(0, t)= \begin{cases}0, & t<0 \\ -1 / M_{0}, & t>0\end{cases}
$$

As in the corresponding piston problem, an expansion fan is again formed by the positive outgoing characteristics from the discontinuity $\xi=t=0$. Hence, the velocity and density are completely determined at $\xi=0$ by the negative and zero-slope characteristics for all times $t>0$. Indeed, we can follow all the steps in the numerical method described above for compressive motion, except that, since $u<0$, the term $u|u|$ in the Fanno model should be replaced by $-u^{2}$ throughout the computations. Figure $11(a, b)$ shows the velocity and pressure solution profiles at different times and for different values of Mach number $M_{0}$.

As in $\S 2$, we again note that none of the expansion waves appear to tend to a travelling wave for large times. This is despite the theoretical possibility that such a travelling wave exists when $\gamma M_{0}=\sqrt{2 \gamma /(\gamma-1)}$ in Ockendon et al. (2001).

In order to investigate this possibility we have used the expansion wave profile in Ockendon et al. (2001) as the initial condition for a Fanno flow on $-\infty<x<\infty$ and 

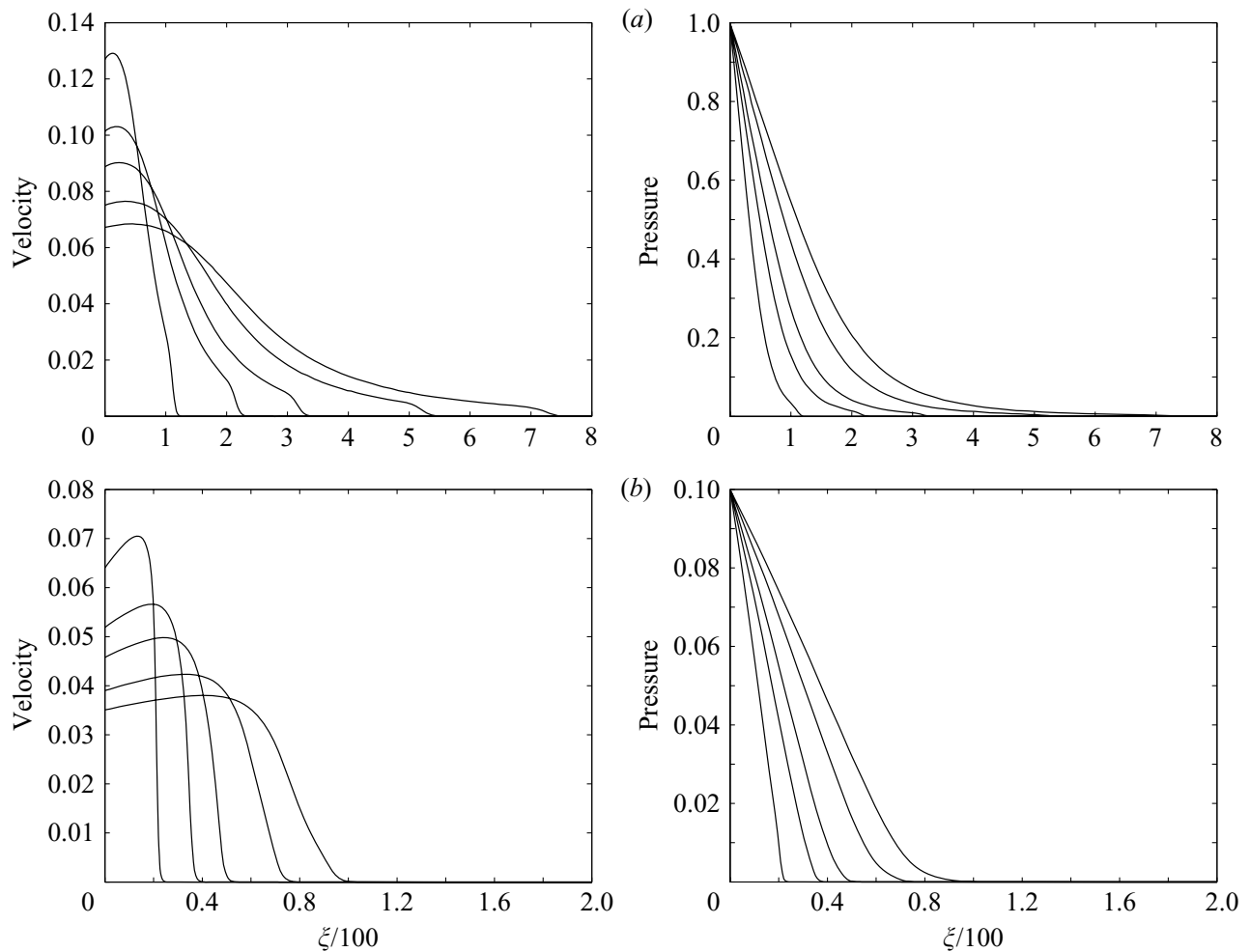

FIGURE 10. Velocity and pressure profiles of the compressive pressure-jump problem at times $t=100,200,300,500,700$ for different Mach number flows: $(a) M_{0}=1,(b) M_{0}=10$.

unphysical oscillations appeared very rapidly for all the time steps we used. Hence, we endorse the conjecture in Ockendon et al. (2001) that such expansion waves are unstable.

\section{Conclusions}

This paper has shown how discretizations based on the method of characteristics can be applied to the Fanno model of compressible pipe flow. We have emphasized the use of the method over long time intervals, so that its predictions can be validated against analytical results for weakly nonlinear flows. In particular, we have considered compression and expansion waves driven by pistons and by step-function pressure changes. Our results suggest that in all compressive flows into a quiescent region, dispersed or partially dispersed shocks will emerge but that they will eventually decay unless energy is continually supplied to the gas. Expansion into a quiescent region is qualitatively different from the predictions of inviscid expansion waves and, even though a travelling wave of expansion is theoretically possible for the Fanno model in a special case, our computations have revealed it to be unstable. Further evidence is provided by figure 12 which displays the velocity profile for the expansion wave produced by a piston withdrawn at speed $\gamma$, corresponding to $M_{0}=\sqrt{2 /(\gamma-1) \gamma}$. Comparison with figure 7 suggests that this value of $M_{0}$ is in no way exceptional.

Our investigations have revealed one interesting open question, namely the evolution of a contact discontinuity under the Fanno model. Preliminary results suggest that it 

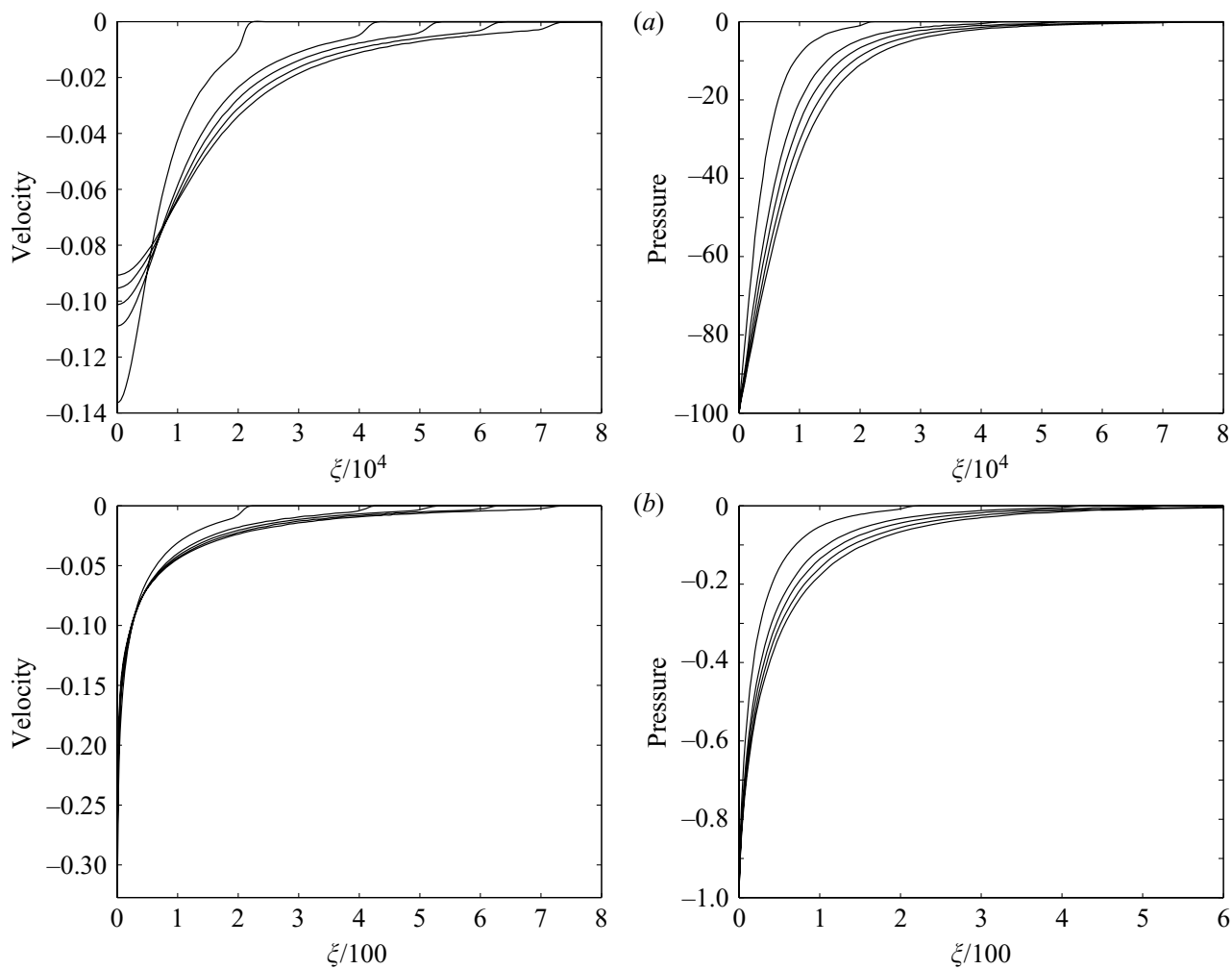

FIGURE 11. Velocity and pressure profiles of the expansive pressure-jump problem at times $t=200,400,500,600,700$ for different Mach number flows: (a) $M_{0}=0.01,(b) M_{0}=1$. Note, as time increases both velocity and pressure profiles get less steep.
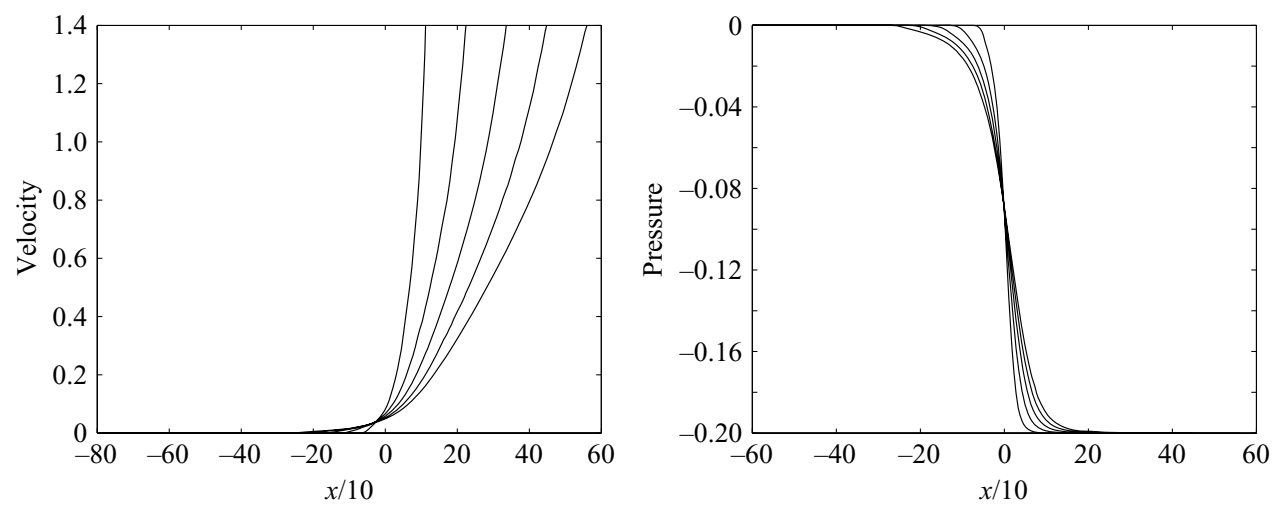

FIGURE 12. Computed velocity and pressure profiles of the expanding piston problem at times $t=80,160,240,320,400$ when $M_{0}=\sqrt{2 /(\gamma-1) \gamma}=1.8898$.

is the speed rather than the strength of contact discontinuities that is most affected by the wall friction.

The authors would like to acknowledge the valuable advice given by P. J. Dellar. Also A. Novikovs was partially supported by the EPSRC and received an Alan Poole scholarship from St Catherine's College, Oxford. 


\section{REFERENCES}

Cebeci, T. \& Smith, A. M. O. 1974 Analysis of Turbulent Boundary Layers. Academic.

Gioia, G. \& Chakraborty, P. 2006 Turbulent friction in rough pipes and the energy spectrum of the phenomenological theory. Phys. Rev. Lett. 96, 044502.

Mathematics In Industry Study Group 2005 University of the Witwatersrand, Piston effect due to cock clllapse, problem description. http://www.wits.ac.za/misgsa2005/.

NikURADSE, J. 1950 Laws of flow in rough pipes. Reprinted in English in NACA Tech. Memo. 1292.

Ockendon, H., Ockendon, J. R. \& Falle, S. A. E. G. 2001 The Fanno model for turbulent compressible flow. J. Fluid Mech. 445, 187-206.

Thompson, K. W. 1987 Time-dependent boundary conditions for hyperbolic systems. J. Comput. Phys. 68(1), 1-24.

Thompson, K. W. 1990 Time-dependent boundary conditions for hyperbolic systems, II. J. Comput. Phys. 89(2), 439-461.

WAGNER, D. H. 1987 Equivalence of the Euler and Lagrangian equations of gas dynamics for weak solutions. J. Diffl Equat. 68, 118-136. 